\title{
基于微孔抽丝成型工艺的软体力/位传感器
}

\author{
姚建涛 ${ }^{1,2}$ 张 弘 ${ }^{1}$ 勾栓栓 $^{1}$ 向喜梅 $^{1}$ 许允斗 $^{1,2}$ 赵永生 $^{1,2}$
}

(1. 燕山大学河北省并联机器人与机电系统实验室 秦皇岛 066004;

2. 先进锻压成形技术与科学教育部重点实验室(燕山大学) 秦皇岛 066004)

\begin{abstract}
摘要: 提出一种全柔性力/位传感器的设计、建模和制造方法, 并进行试验验证。该传感器可通过附着在待测物表面或嵌入其 内部进行力位混合的测量。首先, 创新性地将提出的微孔抽丝成型工艺引入到软体结构的制造中, 构建出低成本、易实现的 软体微通道成型工艺体系, 通过试验比对, 验证了该工艺在软体微通道成型中的优势, 成功实现了全柔性传感器高性能快速 制造。其次, 基于该成型方法设计了双层斜置并行多条的微通道传感器结构, 有效地提高了灵敏度及线性度。最后通过试验 对该传感器的性能进行验证。试验表明该传感器伸长率在 $14 \%$ 以内时, 作为全柔性力传感器线性误差小于 $5 \%$, 灵敏度为 16.95 $\mathrm{mV} / \mathrm{N}$; 作为全柔性位移传感器线性误差小于 $2 \%$, 灵敏度为 $5.56 \mathrm{mV} / \mathrm{mm}$ 。研究内容为全柔性传感器在实际应用中的结构设 计和成型方法中有很大的参考价值。
\end{abstract}

关键词: 全柔性传感器; 力/位感知; 抽丝成型工艺; 液态金属

中图分类号: TH706

\section{Software Force and Displacement Sensor Based on Microporous Wire Drawing Molding Method}

\author{
YAO Jantao $^{1,2}$ ZHANG Hong ${ }^{1}$ GOU Shuanshuan ${ }^{1}$ XIANG Ximei ${ }^{1}$ \\ XU Yundou ${ }^{1,2}$ ZHAO Yongsheng ${ }^{1,2}$ \\ (1. Parallel Robot and Mechatronic System Laboratory of Hebei Province, \\ Yanshan University, Qinhuangdao 066004; \\ 2. Key Laboratory of Advanced Forging \& Stamping Technology and Science, \\ Ministry of Education(Yanshan University), Qinhuangdao 066004)
}

\begin{abstract}
A fully flexible force position sensor's design, modeling and manufacturing method are introduced in this article, and the experimental verification is carried out. The sensor can be used to measure the force or displacement by attaching to the surface of the object or embedding it inside. First of all, the wire drawing molding method of micro manufacturing is introduced into the structure of the software innovatively, construction of low cost, easy to realize the software of micro channel forming process system, verify the advantages of the process in the forming of the micro channel software by comparison, the high performance and rapid manufacturing of the full flexible sensor has been achieved successfully. Secondly, based on the method, a double layer parallel multi strip micro channel sensor structure is designed, which effectively improves the sensitivity and linearity. The performance of the sensor has been verified through experiments. When the elongation is less than $14 \%$, as a full flexible force sensor, the linear error is less than $5 \%$, and the sensitivity is $16.95 \mathrm{mV} / \mathrm{N}$. As a full flexible displacement sensor, the linear error is less than $2 \%$, and the sensitivity is 5.56 $\mathrm{mV} / \mathrm{mm}$. The research content has great reference value for the structure design and forming method of the full flexible sensor in practical application.
\end{abstract}

Key words: fully flexible sensor; force or displacement perception; wire drawing molding method; liquid metal

\section{0 前言}

近年来，智能穿戴、康复医疗等应用领域伴随

20180402 收到初稿, 20180919 收到修改稿
着柔性机器人技术的发展逐渐地进入了人们的视野 之中 ${ }^{[1-3]}$ 。在共融机器人技术的研究中, 着重于人体 在交互过程中的体感及交互的安全性是很有必要 的, 其中, 柔性传感器技术将发挥着重要的作用。 在传统的传感器的研究中, 分为刚性传感器和柔性 传感器, 柔性传感器主要以小柔性变形为主, 整体 
仍为刚性结构, 并不能像全柔性传感器那样去抵抗 外部环境的冲击, 人体佩戴时的舒适感也较差, 使 其在智能穿戴等领域的应用受限 ${ }^{[4-8]}$ 。此外, 一些大 柔性传感器中仍使用着硬质敏感元件, 在使用中遭 受到外部大力冲击时也会对柔性基体及敏感元件 造成损坏, 不安全的同时, 也会影响传感器的使用

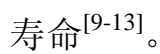

为了彻底消除力传感器的硬质部分, 基于新材 料技术的全柔性传感器已成为目前研究的前沿课 题。研究人员为了研制出具有良好延展性的力传感 器, 通过新材料性能的探讨研究, 结合传感器技术, 开发出了一系列超弹性力传感器, 用于智能穿戴设 备的研究, 并进行了相应的试验研究, 体现了大变 形全柔性传感器应用在智能穿戴中的优越性 ${ }^{[14-18]}$ 。

在软体中成型微通道并加入液态敏感元件的传 感器制造方法具有良好的性能优势, 国际上大量研 究团队进行了相关方面的前沿探索。CHOI 等 ${ }^{[19]}$ 在 柔性基体 Ecoflex 内部的通道中加入 $\mathrm{EG} / \mathrm{NaCl}$ 离子 溶液, 进行传感器性能方面的研究, 研制出具有高 延展率, 低滞后性的力传感器。RUSSO 等 ${ }^{[20]}$ 在硅 橡胶中注入与人体相容的 $\mathrm{NaCl}$ 离子溶液, 研制出 可延展的软体传感器, 该传感器在 $64 \%$ 以内的延展 率下具有良好的线性度。

哈佛大学通过将炭黑颗粒混入至硅酮油中, 作 为一种功能性墨水作为柔性敏感材料, 并通过内部 3D 打印技术在柔性基体中成型出微通道, 将液态敏 感元件注入至该微通道中, 制成全柔性传感器。当 传感器受力时, 由于基体变形, 内部微通道的尺寸 会发生一定的变化, 进而导致内部敏感元件的阻值 发生变化, 通过检测敏感元件阻值的变化, 来判断 力的大小。此外, 该团队基于这种传感器制造方法, 将液态合金嵌入到微通道中进行单维及多维力的检 测 $[21-26]$ 。

在国内, PVDF 以其良好的柔性及轻质的重量 并且易加工、稳定性好等优点, 被广泛应用于力传 感器的设计制造中, 研究人员从多种角度对其进行 分析研究 ${ }^{[27-28]}$, 制造出基于 PVDF 压电薄膜的一系 列力传感器。大连理工大学从传感器构型方面入手, 设计了一种多曲面的 PVDF 力传感器, 其中以硅胶 棒为基体来增强传感器的整体柔性 ${ }^{[29]}$ 。电容式柔 性压力传感器在国内也有一定的研究 ${ }^{[30]}$, 翟庆彬 等以 PDMS 为柔性基体, 通过研究不同材料为电 极及介电层, 进行了常用电容式传感器的性能分 析 $^{[31]}$ 。北京印刷学院以纳米银线为导电材料, PDMS 为弹性基体, 设计了柔性压力传感器与拉力 传感器 ${ }^{[2-33]}$ 。
在全柔性传感器的制作上, 各有千秋, 其微通 道成型是主要难度和关键因素。成型方法普遍采用 多层叠加思想, 即通过模子成型出单层弹性体后, 再通过粘接叠加的方式将各个形状的单层体合成一 个整体，层间的间隙即为内部微通道的形状，这类 设计方法可以成型绝大多数较为复杂的微通道。但 此类思想的成型过程中, 难免因操作不当而导致微 孔壁厚不均匀，甚至堵塞微孔，且在沉积液态金属 敏感元件时, 不可避免的会在层间沾有金属残留液, 导致其材料粘接面粘接不可靠, 粘和的密封性不好, 最终体现在不良的耐受性能, 长期使用下不可避免 的会出现层间开裂现象。相比之下, 采用冷冻切割 成型技术成型金属形体形态，再进行叠加封装的方 法做出的传感器精度更高, 其稳定性能更可靠。但 总的来说，此类上下分层叠加的制作思路难以克服 微腔成型精度不高, 成型后传感器性能不高的缺点。 此外 3D 打印技术成型微通道 ${ }^{[34-35]}$, 相比其余制备 工艺, 其成型速度快, 制备效率高, 适合工业量产 与个性化定制, 但其前期投入成本过高。

3D 打印成型方法, 设备昂贵, 而传统的模具成 型工艺, 微通道成型精度较差。针对这些问题, 本 文提出独特的抽丝成型方法进行微通道成型。此外, 本文从微通道的构型角度进行柔性传感器主要性能 的分析, 研制出高灵敏度及良好线性度的全柔性传 感器。

\section{1 设计原理及数学模型}

本文设计了一种具有双层、空间布局的软体内 部微通道结构, 并基于在微通道中通入液态合金的 方法, 进行力位混合测量。图 1 所示, 传感器可置

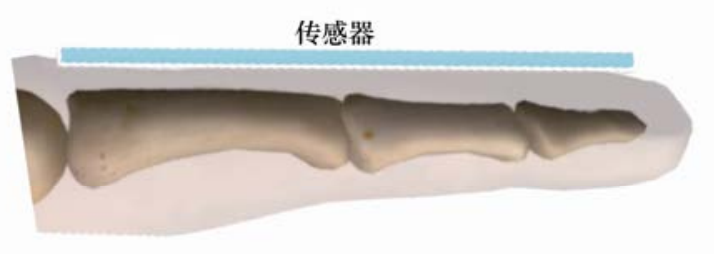

(a) 传感器用于指背力 / 位测量

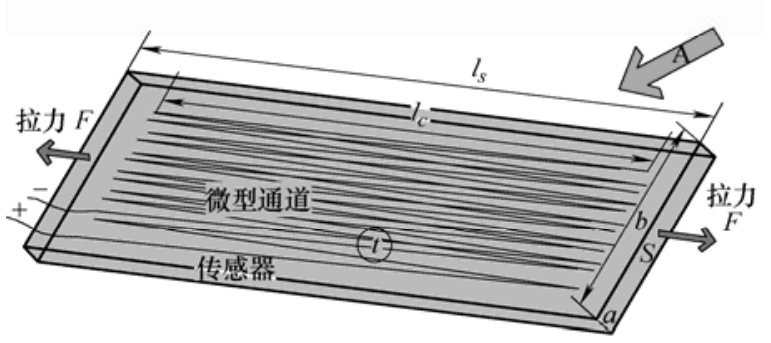

(b) 传感器整体结构构型图 


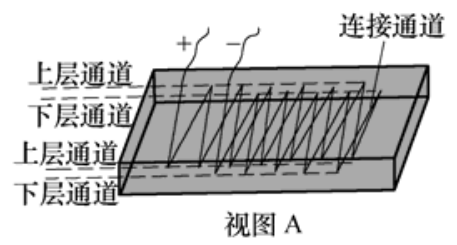

(c) 传感器内部微通道构型图

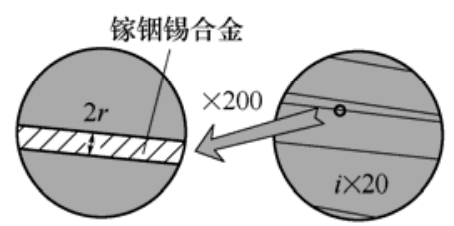

(d) 微通道内部放大示意图

图 1 传感器结构示意图

于手指或其他关节背处, 内部成型出双层微型通道, 在空间上以层间错路的方式进行布置。当传感器拉 伸时, 微通道内部的液态合金电阻发生变化, 通过 对电阻变化的测量, 结合超弹性材料的力位性能, 可得到传感器在拉伸时力及位移的情况。

液态金属被完全密封在软体微通道中，当传感 器受到拉力时, 软体微通道产生变形, 但由于液态 金属为不可压缩材料, 其随着微通道的变形, 总体 积不发生变化，即

$$
\int_{0}^{l_{\mathrm{c} 0}} f_{\mathrm{A}_{\mathrm{c} 0}}(x) \mathrm{d} x=\int_{0}^{l_{\mathrm{c}}} f_{\mathrm{A}_{\mathrm{c}}}(x) \mathrm{d} x
$$

式中, $l_{\mathrm{c} 0}$ 与 $l_{\mathrm{c}}$ 分别为传感器受力变形前后微通道的总 长度, $f_{\mathrm{A}_{\mathrm{t}}}(x)$ 与 $f_{\mathrm{A}_{\mathrm{c}}}(x)$ 分别为传感器受力变形前后微 通道的截面积。微通道中液态金属的总电阻 $R_{\mathrm{c}}$ 为

$$
R_{\mathrm{c}}=\int_{0}^{l_{\mathrm{c}}} \rho \frac{1}{f_{\mathrm{A}_{\mathrm{c}}}(x)} \mathrm{d} x
$$

由于传感器中微通道的直径很小, 仅为微米级 别, 可将微通道假设为恒定直径的圆柱形微通道。 简化整理公式可得

$$
R_{\mathrm{c}}=R_{\mathrm{c} 0}\left(\frac{l_{\mathrm{c}}}{l_{\mathrm{c} 0}}\right)^{2}
$$

式中, $R_{\mathrm{C} 0}$ 为微通道中液态金属的初始电阻值。此外, 考虑全柔性传感器的测量性能, 导出传感器受力时 电阻变化与应变之间的关系 ${ }^{[36]}$

$$
\frac{\Delta R}{R_{\mathrm{c} 0}}=\varepsilon^{2}+2 \varepsilon
$$

从式(4)中可以看出, 全柔性传感器的电阻与应 变之间的关系与传感器本体的结构等因素并没有直 接的关系。所以传感器的灵敏度主要取决于传感器 内部电阻和外部电路的电阻的关系上。由于传感器 在实际应用中, 需要考虑外接电路对传感器的影响, 电阻变化信号测量时, 外接电路电阻 $R_{e}$ 会对其造成
一定的影响。即

$$
\frac{\Delta R}{R_{0}}=\left(\varepsilon^{2}+2 \varepsilon\right) \frac{R_{\mathrm{c} 0}}{R_{\mathrm{c} 0}+R_{e}}
$$

式中, $R_{0}$ 为传感器测量外部电路的电阻值。由式(5) 可以得出位移与电阻变化量的关系, 并得出为提高 传感器的灵敏度, 需要提高传感器中敏感元件的阻 值, 从构型的角度来看, 可采用两种方法: 提高微 通道的总长度; 降低微通道的横截面积。

此外, 可将具有长条状柔性硅橡胶的弹性体简 化为一个弹性模量 $E$ 的微变弹簧, 近似满足胡克定 律, 可得两端拉力

$$
F=E S \varepsilon
$$

式中 $E$ 一一柔性硅橡胶的弹性模量;

$S$ 一一传感器纵向横截面积。

硅橡胶内部微腔极为微小, 即使通道内液态合 金处于充盈形态，其对整体硅橡胶的力学性能影响 很小。在硅橡胶拉伸时, 可假设将硅橡胶看作各 向同性良好的弹性体，横向收缩应变与纵向伸长 应变满足泊松提出的关系式, 因此传感器横截面 面积

$$
S=a b(1-v \varepsilon)^{2}
$$

式中 $a$-一横截面的长:

$B$ 一一横截面的宽;

$V$ ——硅橡胶伯松比。

则有

$$
F=E a b(1-v \varepsilon)^{2} \varepsilon
$$

通过联立式(5)、(8)可得到力与电阻变化量的关 系, 结合超弹性材料的应力应变性质, 可以看出按 力传感器进行测量时, 灵敏度指标与基体材料的弹 性模量及传感器的横截面积有关, 即弹性模量越低、 横截面积越小, 传感器的灵敏度越高。而在硫化条 件完全相同的情况下成型的相同性能的硅胶，常温 下其弹性模量稳定。因此, 在使用同样传感器基体 材料以及同样外形尺寸的传感器中, 研究传感器中 微通道的直径及分布非常重要。因此，本文中采用 空间双层布置传感器微通道的方法, 来提高力位传 感器的灵敏度。

\section{2 微孔抽丝成型工艺}

为提高传感器性能, 创新性地提出了软体微通道 抽丝成型工艺，通过该工艺，既可保证成型出微通道 的精度，又可用于多条多层多种布局的微通道的成 型。该方法采用高精度细金属线作为微通道的铸造内 
芯模具, 与目前的微通道成型工艺相比, 该工艺可一 次成型出微通道, 保证了微通道的精度及力学性能。

以成型该柔性力/位传感器的过程为例, 全柔性 力/位硅橡胶传感器其内部的通道为双层往返式微 腔, 层间在空间上以交错连接的方式布置, 为典型 的复杂空腔构造, 其结构极其精巧细微, 微腔半径 小至 $0.035 \mathrm{~mm}$, 且为回型空腔, 其加工成型工艺难 度系数大, 需要考虑的因素较多。为保证传感器的 整体成型, 该工艺采用分段制作分次成型思想。可 细分为三个阶段, 先成型核心微腔段, 后成型微腔 头尾段, 最后注入镓铟锡合金。下文对该工艺整体 进行详细介绍。

该传感器使用的室温硫化型硅橡胶(RTV-2), 分 为 $A 、 B$ 组份, 一比一配合后, 可在 $80{ }^{\circ} \mathrm{C}$ 下, $10 \mathrm{~min}$ 内硫化, 呈现出高聚物的高弹性。具体成型工艺步 骤如图 2 所示。

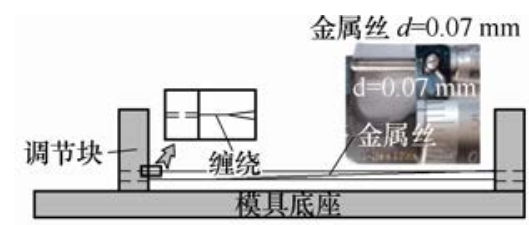

(a) 精密铜线布置示意图

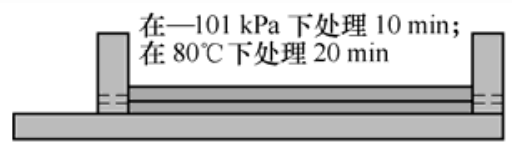

(b) 除气泡及保温凝固

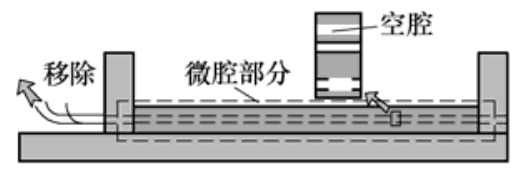

(c) 抽除基体内部铜线

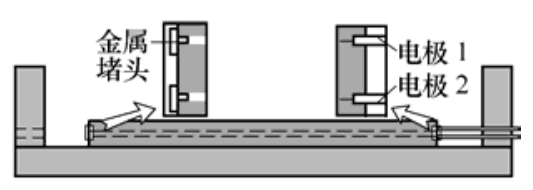

(d) 封闭微通道置入电极

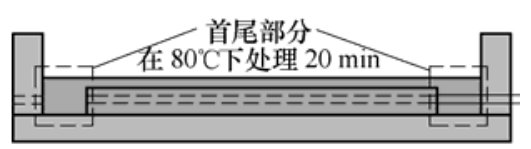

(e) 浇注硅胶封闭两端

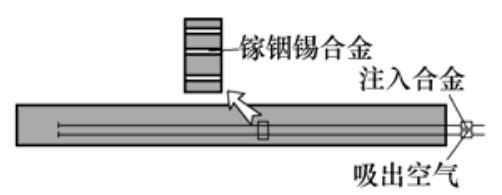

(f) 注入镓铟锡合金

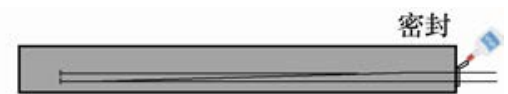

(g) 柔性胶完成密封

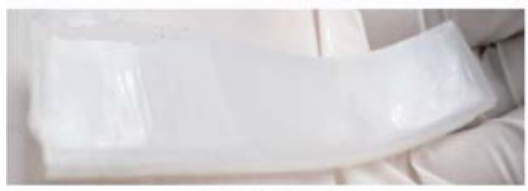

(h) 成型后的传感器

图 2 双层全柔性力/位传感器成型工艺流程

首先成型微腔段, 微腔段的成型主要采用精密 紫铜金属线作为内芯模, 成型出与内芯模直径基本 相同的微通道主腔, 具体步骤如下: 使用由高精度 3D 打印加工成型的模具(具有模具底座和滑动调节 块), 按照图 2a 中层间错落的微腔通道走线方式在 模具中拉扯直径 $0.07 \pm 0.01 \mathrm{~mm}$ 的紫铜金属线, 且 在相邻两金属线连接处将金属线缠绕一小段距离, 在浇筑成型后, 缠绕的金属段将作为连通相邻通道 的转接段。将柔性硅橡胶溶液一比一调配充分摚拌 均匀后, 置于-101 kPa 环境下 $5 \mathrm{~min}$, 充分去除内 部空气, 而后浇筑至模中, 并放置于 $80{ }^{\circ} \mathrm{C}$ 下 $10 \mathrm{~min}$, 如图 $2 \mathrm{~b}$ 所示。在室温冷却 $10 \mathrm{~min}$ 后, 抽离内部金 属丝, 其中, 丝线所通之处即为微腔, 如图 2c 所示。

成型首尾段, 如图 2d、2e 所示, 成型首尾段的 主要目的在于对微腔的两端进行密封。在两根金属 线末端缠绕处抽离形成的洞口嵌入长度不长于缠绕 距离的金属铜柱, 留出一定空间, 防止再次浇注时 堵塞微腔, 使得两条微腔连成一个连通的折返通道, 同时在微腔首尾接入正负极引线。调节滑动调节块 增大成型腔, 再次浇注硅橡胶, 置于 $80{ }^{\circ} \mathrm{C}$, 保温 $10 \mathrm{~min}$ 。

注入镓铟锡合金, 如图 $2 \mathrm{f}$ 所示, 在成型出的微 通道中注入液态金属镓铟锡合金作为传感器的敏感 元件。采用微型注射器, 从一端引线端紧挨引线平行 插入注射针头, 加压将镓铟锡合金注入, 另一端引线 端则插入针头抽离空气, 使得两端引线电阻达到预定 值。用硅胶专用胶水密封注射插口, 整体封装。

如图 2h 所示为成型出的传感器的外观图, 进行 局部修正, 剔除毛刺、瑕疵等不光整缺陷, 使得外 表光洁整齐。如图 3 为双层全柔性力/位传感器整体 展示(传感器微通道分布细节展示; 传感器可随意扭 曲拉伸; 传感器可随意弯曲), 可以看出, 由该方法 成型出的传感器为一个完备的整体, 其内部微型通 道相互连通。此外, 该成型工艺绿色环保, 成本低, 精度高, 可清晰地看到内部通道排列极为有序, 分 为上下双层, 层间相互错开, 每层相邻通道为 $\mathrm{Z}$ 字 
形连接。整体极其柔软, 即使在扭曲的情况下, 拉 伸率可达 $180 \%$ 。与传统的上下分层成型再粘合的 成型方法相比, 此工艺成型出的细微通道是一体化 成型的整体, 通道尺寸更加准确, 内壁更加光滑精 密, 性能更加可靠耐用, 通道内甚至能承受高压态 液体。此外, 简单地更换不同直径的金属丝即可调 整微通道直径, 通过调整穿线孔位置和改变穿线方 式即可调整微通道的形状。
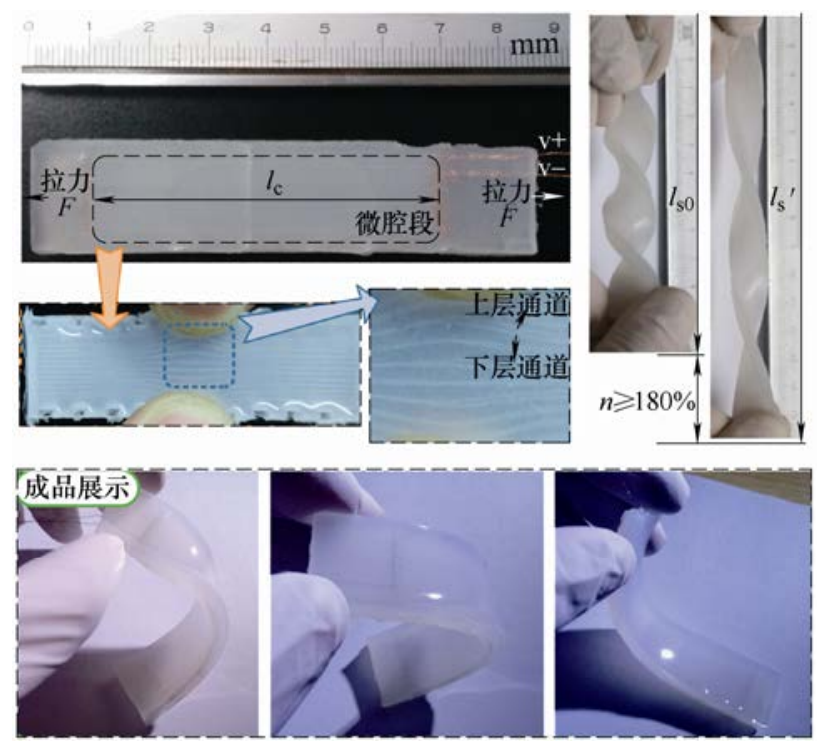

图 3 双层全柔性力位混合传感器整体展示

\section{3 标定试验及性能测试}

基于上述的微孔抽丝成型工艺, 设计制造出了 柔性力/位传感器, 如图 3 所示。通过微孔抽丝成型 工艺, 成型出两层微通道, 每层通道 $\mathrm{Z}$ 字形排布连 通, 上层为 13 条通道, 通过一根过渡通道与下层 10 条 $Z$ 字形通道连接, 上下层末端引出正负极导线。 其中, 每相邻通道间夹角为 $\theta=2 \times \arctan 1 / 63=$ $1.81876^{\circ}$, 通道半径 $r$ 为 $0.035 \mathrm{~mm}$, 取 $b=4 \mathrm{~mm}$ 、 $a=18 \mathrm{~mm} 、 l_{\mathrm{s}}=73 \mathrm{~mm} 、 l_{\mathrm{c}}=35 \mathrm{~mm}$ 。在通道内注入低 共熔点镓铟锡合金, 其 $-19{ }^{\circ} \mathrm{C}$ 的熔点使得在常温下 $\left(18 \sim 25{ }^{\circ} \mathrm{C}\right)$ 能良好的工作。当在两端施加拉力 $F$ 使 柔性硅橡胶发生拉伸形变时, 其内的金属溶液随微 型通道发生同步拉伸变化。

图 4 为佩戴在食指上的全柔性力/位传感器的力 电压标定曲线, 经拟合处理, 该传感器灵敏度为 $16.95 \mathrm{mV} / \mathrm{N}$, 在 $14 \%$ 的延伸率内, 线性度为 $4.593 \%$ 。

实际恒流源值为 $8.257 \mathrm{~mA}$, 当拉伸率为 $14 \%$ 时, 电压增加至 $0.0565 \mathrm{~V}$, 对应最大拉力 $3.33 \mathrm{~N}$ 。

实际恒流源值为 $8.257 \mathrm{~mA}$, 当拉伸率为 $14 \%$ 时, 电压增加至 $0.0565 \mathrm{~V}$, 对应最大拉伸量 10.17 $\mathrm{mm}$ 。

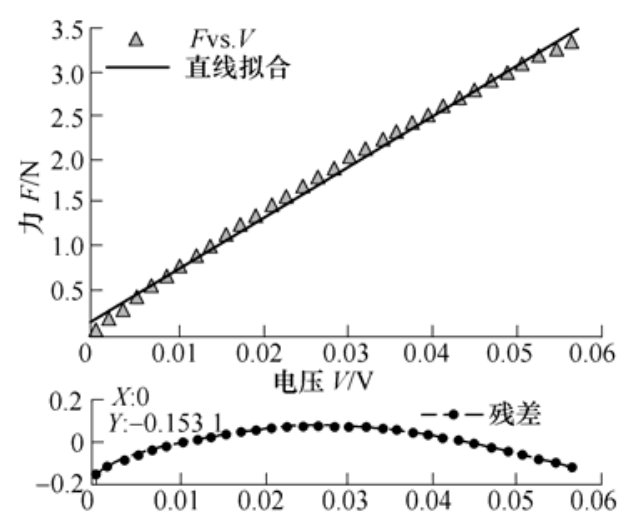

(a) 直线拟合

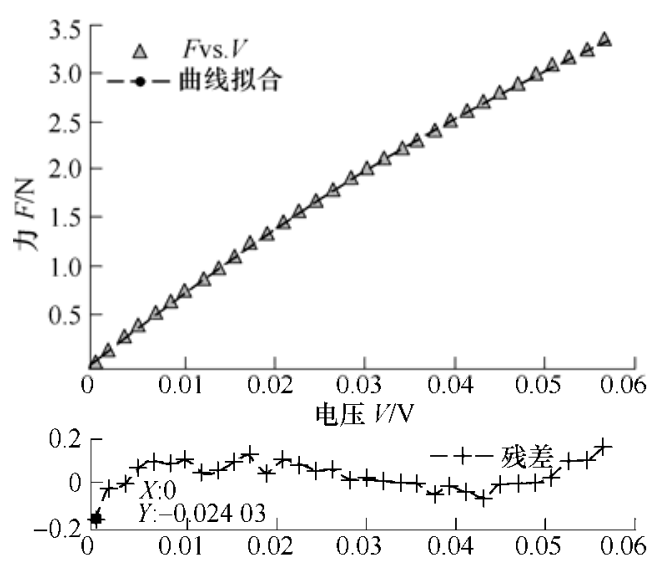

(b) 曲线拟合

图 4 传感器电压与 $V 、 F$ 拟合曲线

拉力与电压拟合, 如图 4 所示, 三角形为电压 与拉力的试验数据, 力随着电压增大, 其增长的趋 势趋于缓慢, 与上文理论所述相吻合。如图 4a 所示, 在进行线性拟合时, 在线性度上, 其和方差 SSE 为 0.1425 , 曲线拟合确定系数 R-square 为 0.9955 , 线 性度为 $\pm 4.593 \%$ F.S, 基本呈线性关系。图 4b 为曲 线拟合曲线, 其拟合效果接近理想, 曲线拟合确定 系数 R-square 高达 0.9999339 , 其和方差 SSE 为 0.002084 , 最大误差为 $0.7216 \%$, 灵敏度为 16.95 $\mathrm{mV} / \mathrm{N}$ 。

位移与电压拟合如图 5a 所示, 绿色圆为电压与 位移的试验数据, 其位移 $\Delta L_{s}(\max )$ 为 $10.17 \mathrm{~mm}$, 其 线性度为 $\pm 1.734 \%$ F.S。拟合误差同样为抛物线形 式, 曲线拟合确定系数 R-square 为 0.9955 , 较力与 电压误差小, 与上文理论所述相吻合。而图 5b 用曲 线拟合时，其曲线拟合确定系数 R-square 为 0.9999584 , 最大误差仅为 $0.4043 \%$, 灵敏度为 5.56 $\mathrm{mV} / \mathrm{mm}$ 。

标定试验的结果, 拉力与电压的直线拟合误差 为 $5 \%$ 以内, 而曲线拟合误差为 $0.7 \%$ 。位移与电压 的直线拟合误差为 $2 \%$ 以内, 曲线拟合误差仅为 $0.4 \%$ 。此精度在柔性力测量场合, 有较好应用前景。 

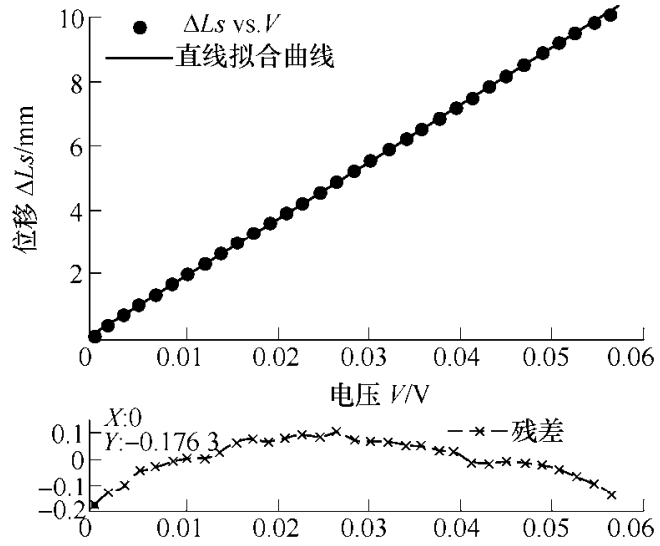

(a) 直线拟合
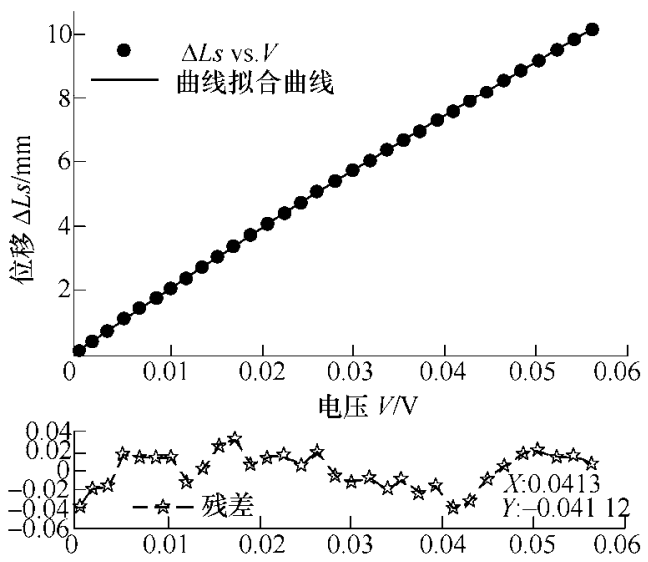

(b) 曲线拟合

图 5 传感器电压与 $V 、 \Delta L_{s}$ 拟合曲线

\section{4 性能对比及迟滞性分析}

为了更准确地对微孔抽丝成型柔性传感器的方 法与其它成型方法进行对比, 采用和另一种倒模工 艺 $^{[37]}$ 成型方法进行对比。倒模工艺过程是通过模具 成型出具有凸凹微通道形貌的单层弹性体, 再通过 粘接叠加方式将单层弹性体合成一个整体, 层间形 成的通道即为内部微通道。试验中采用相同外形大 小的传感器, 并内置相同数目长度的微通道, 根据 工艺特点分别采用横截面积大小相同的长方形及圆 形状横截面的微通道, 且内部通入相同体积的液态 合金，保证初始的电阻值相同。

通过对两种成型传感器的拉伸测试试验, 得到 位移标定曲线和力标定试验。图 6a 为倒模工艺制造 传感器的应变与电阻变化量的关系曲线, 其线性度 为 $7.89 \%$ 、迟滞误差为 $10.54 \%$ 。图 6b 为抽丝工艺 制造传感器的应变与电阻变化量的关系曲线, 其线 性度为 3.08\%, 迟滞误差为 $0.36 \%$ 。图 6c 为倒模工 艺制造传感器的受力与电阻变化量的关系曲线, 其 线性度为 $15.7 \%$, 迟滞误差为 $20 \%$ 。图 6d 为抽丝工

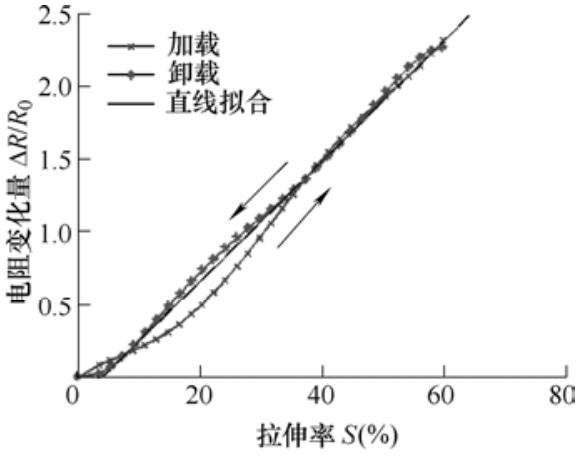

(a) 倒模工艺制造传感器的位移标定曲线

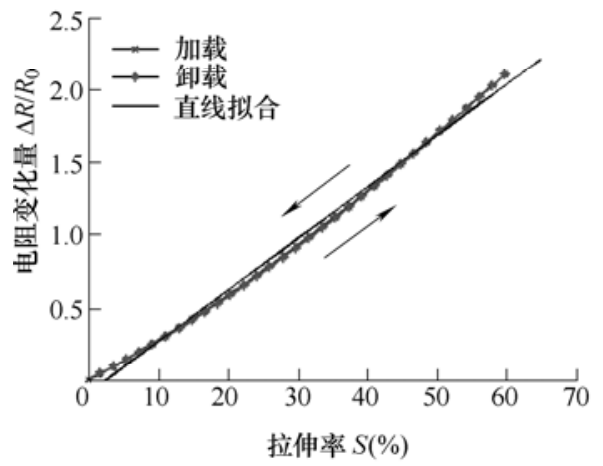

(b) 抽丝工艺制造传感器的位移标定曲线

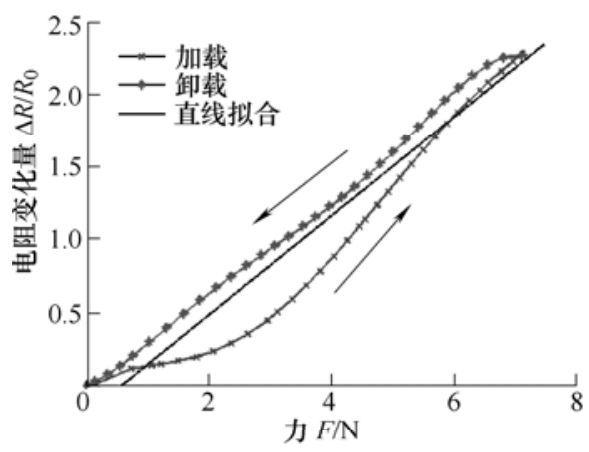

(c) 倒模工艺制造传感器的位移标定曲线

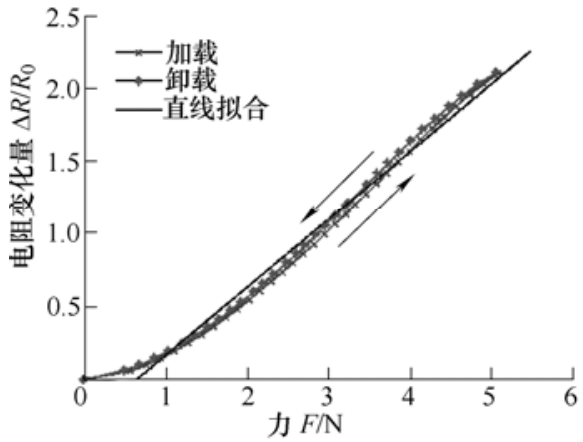

(d) 抽丝工艺制造传感器的力标定曲线

图 6 传感器标定曲线

艺制造传感器的受力与电阻变化量的关系曲线, 其 线性度为 4.89\%、迟滞误差为 3.2\%。对比两者可得 出基于微孔抽丝成型工艺的力/位传感器的线性度 及迟滞误差都略优于基于倒模工艺成型的力/位传 感器。由于高弹性材料的粘弹性特性, 传感器的受 力与位移关系曲线中能够明显看出具有较大的迟滞 
误差及非线性误差。而该传感器的电阻变化量与应 变的标定曲线中的迟滞误差可以忽略不计, 非线性 误差也较低。

由于在实际应用中, 超弹性材料与理想弹性固 体及理想流体均不同, 而是组合了固体的弹性和液 体的粘性两者的特征, 即粘弹性。受到分子链形态、 结构等因素的影响, 其弹性模量并不是一个常量, 而是与时间相关的变量, 力学性质也随着时间变化, 从而产生力学松弛现象, 力学松弛包括静态的粘弹 性(蠕变、应力松弛)和动态的粘弹性(滞后现象、力 学损耗)。受高弹性材料力学松弛现象的影响, 在高 弹性材料的应力应变曲线中, 加载与卸载过程位移 和载荷总相差某个相位差。

在加载卸载过程中, 除了应力应变存在滞后现 象, 传感器的应变与电阻变化之间的关系也存在滞 后现象。目前, 在由超弹性材料制成的各类应变传 感器中, 迟滞误差均大于 $2 \%$, 其主要原因是超弹 性材料在变形时的应力松弛决定的, 应力松弛为在 恒定的温度和形变不变的情况下, 聚合物内部应力 随着时间的增长而逐渐衰弱的现象。由于高弹性材 料被拉长时, 处于不平衡构象, 要逐渐过渡到平衡 的构象, 即链段随着外力的方向运动以减小或者消 除内部应力, 如果温度很高, 链运动摩擦阻力很小, 应力很快弛掉了, 所以观察不到, 反之, 内摩擦阻 力很大, 链段运动能力差, 应力松驰慢, 也观察不 到, 只有在 $T_{g}$ (玻璃转化点)温度附近的几十度的范 围内应力松驰现象比较明显。

在传感器拉伸过程中, 硅橡胶横向收缩, 挤压 微通道中的液态金属向两端流动, 最终保持微通道 初步的受力平衡状态, 当传感器均采用同一种液态 金属时, 该平衡时间与微通道的所受到的挤压力有 直接关系, 当微通道的受力越大时, 传感器初步达 到平衡状态时的时间越短。因此, 为了改善传感器 的动态性能, 提出在微通道中通入过量液态敏感元 件的方法, 即在微通道中通入液态敏感元件的体积 大于微通道的体积。

通过该方法可使液态敏感元件对微通道内壁始 终产生一定的膨胀力 $F_{i}$, 初始状态下, 膨胀的微通 道对液态敏感元件的挤压力为 $F_{c}=F_{i}$, 在硅橡胶横 向收缩时, 挤压力 $F_{c}$ 变大, 压缩液态敏感元件流动 到平衡位置。假设该液态敏感元件为理想流体, 在 流动时各层之间没有相互作用的切应力, 高膨胀率 会产生大的挤压力, 会加速液态敏感元件达到稳定 状态。

此外, 由于超弹性材料应力松弛现象, 聚合物 内部应力逐渐减小, 材料的泊松比增加, 微通道横
截面积增大, 从而导致检测到敏感元件的阻值减小, 影响传感器使用的稳定性。改善超弹性材料应力松 弛的基本方法为提高材料内部链运动摩擦阻力, 提 高应力的松驰速度。这样, 当液态敏感元件的膨胀 力足够大时, 可有效改善硅胶内部的应力松驰现象, 加速应力松弛, 而由于膨胀力远大于松弛掉的应力, 对微通道体积的影响不大, 可以忽略不计。上述试 验中采用的微孔抽丝工艺采用了内置膨胀液态合金 的方法, 便有效的改变了传感器的迟滞性。

\section{5 结论}

(1) 建立了全柔性力位传感器的数学模型, 得 出通过提高微通道总长、降低微通道横截面面积两 种方法可有效提高全柔性传感器灵敏度, 并在此基 础上, 提出多层多条 $Z$ 字型通道构型, 以提高传感 器性能。

(2) 创新性地提出微孔抽丝成型工艺。该工艺 降低了生产门槛, 成本低, 易于调整参数。

（3）试验以及性能对比分析表明, 微孔抽丝成 型工艺成型的柔性力/位传感器在线性度及迟滞误 差方面均具有良好的性能。该传感器在柔性力测量 方面有广阔的应用前景。

\section{参 考 文 献}

[1] 费燕琼, 庞武, 于文博. 气压驱动软体机器人运动研究 [J]. 机械工程学报, 2017, 53(17): 14-18.

FEI Yanqiong, PANG Wu, YU Wenbo. Movement of air-driven soft robot[J]. Journal of Mechanical Engineering, 2017, 53(17): 14-18.

[2] 张成新, 余跃庆. 柔性机器人协调操作的动力学建模与 分析 [J]. 机械工程学报, 2003, 39(5): 49-54.

ZHANG Chengxin, YU Yueqing. Dynamic modeling and analysis for the cooperation of flexible manipulators[J]. Journal of Mechanical Engineering, 2003, 39(5): 49-54.

[3] 于靖军, 毕树生, 宗光华. 全柔性机器人机构结构动力 学分析方法研究[J]. 机械工程学报, 2004, 40(8): 54-58 YU Jingjun, BI Shusheng, ZONG Guanghua. Research on structural dynamics of a general fully compliant robotic mechanism[J]. Journal of Mechanical Engineering, 2004, 40(8): $54-58$

[4] BEEBE D J, HSIEH A S, DENTON D D, et al. A silicon force sensor for robotics and medicine[J]. Sensors \& Actuators A Physical, 1995, 50(1-2): 55-65.

[5] 马丙辉, 卢泽生. 小型微力传感器的研制[J]. 机械工程 学报, 2006, 42: 227-230

MA Binghui, LU Zesheng. Development of micro force 
sensor[J]. Journal of Mechanical Engineering, 2006, 42: 227-230.

[6] 谢石林, 陈胜来, 张希农, 等. 传感器标定的神经网络 杂交建模方法[J]. 机械工程学报, 2010, 46(22): 6-15 XIE Shilin, CHEN Shenglai, ZHANG Xinong, et al. Neural network hybrid modeling method for transducer calibration[J]. Journal of Mechanical Engineering, 2010, 46(22): 6-15

[7] 王宣银, 尹瑞多. 基于 Stewart 机构的六维力/力矩传感 器[J]. 机械工程学报, 2008, 44(12): 118-122.

WANG Xuanyin, YIN Ruiduo. Six-axis force/torque sensor based on stewart platform[J]. Journal of Mechanical Engineering, 2008, 44(12): 118-122.

[8] YAO J, ZHANG $\mathrm{H}$, XIANG X, et al. A 3-D printed redundant six-component force sensor with eight parallel limbs[J]. Sensors \& Actuators A Physical, 2016, 247: 90-97.

[9] LACOUR S P, JONES J, WAGNER S, et al. Stretchable Interconnects for Elastic Electronic Surfaces[J]. Proceedings of the IEEE, 2005, 93(8): 1459-1467.

[10] KIM D H, SONG J, CHOI W M, et al. Materials and noncoplanar mesh designs for integrated circuits with linear elastic responses to extreme mechanical deformations[J]. Proceedings of the National Academy of Sciences of the United States of America, 2008, 105(48): 18675-18680.

[11] ADREGA T, LACOUR S P. Stretchable gold conductors embedded in PDMS and patterned by photolithography: Fabrication and electromechanical characterization[J]. Journal of Micromechanics \& Microengineering, 2010, 20(5): 055025.

[12] WETTELS N, SANTOS V J. Biomimetic tactile sensor array[J]. Advanced Robotics, 2008, 22(8): 829-849.

[13] KUMAR N, PICCIN O, MEYLHEUC L, et al. Design and modeling of a polymer force sensor[J]. IEEE/ASME Transactions on Mechatronics, 2016, 21(1): 555-564.

[14] PARK J J, HYUN W J, MUN S C, et al. Highly stretchable and wearable graphene strain sensors with controllable sensitivity for human motion monitoring[J]. Acs Applied Materials \& Interfaces, 2015, 7(11): 6317-6324.

[15] YOO N S G, KOO H J, CHANG S T. Highly stretchable and transparent microfluidic strain sensors for monitoring human body motions[J]. Acs Applied Materials \& Interfaces, 2015, 7(49): 27562-27570.

[16] RADHA B, SAGADE A A, KULKARNI G U. Flexible and semitransparent strain sensors based on micromolded Pd nanoparticle-carbon $\mu$-stripes[J]. Acs Applied Materials \& Interfaces, 2011，3(7): 2173-2178.
[17] TAN G Y, ZHAO Z, HU H, et al. Highly stretchable and ultrasensitive strain sensor based on reduced graphene oxide microtubes-elastomer composite[J]. Acs Applied Materials \& Interfaces, 2015， 7(49): 27432-27439.

[18] HARRIS K D, ELIAS A L, CHUNG H J. Flexible electronics under strain : A review of mechanical characterization and durability enhancement strategies[J]. Journal of Materials Science, 2016, 51(6): 2771-2805.

[19] CHOI D Y, KIM M H, OH Y S, et al. Highly stretchable, hysteresis-free ionic liquid-based strain sensor for precise human motion monitoring[J]. Acs Applied Materials \& Interfaces, 2017, 9: 1770-1780.

[20] RUSSO S, RANZANI T, LIU H, et al. Soft and stretchable sensor using biocompatible electrodes and liquid for medical applications[J]. Soft Robotics, 2015, 2(4): 146-154.

[21] AMJADI M, KYUNG K, PARK I, et al. Stretchable, skin-mountable, and wearable strain sensors and their potential applications: A review[J]. Advanced Functional Materials, 2015, 26(11): 1678-1698.

[22] KIM H J, SON C, ZIAIE B. A multiaxial stretchable interconnect using liquid-alloy-filled elastomeric microchannels[J]. Applied Physics Letters, 2008, 92: 011904.

[23] PARK Y L, MAJIDI C, KRAMER R, et al. Hyperelastic pressure sensing with a liquid-embedded elastomer[J]. Journal of Micromechanics \& Microengineering, 2010, 20(12): 125029-125034(6).

[24] FASSLER A, MAJIDI C. 3D structures of liquid-phase GaIn alloy embedded in PDMS with freeze casting[J]. Lab on A Chip, 2013, 13(22): 4442-4450.

[25] CUMBY B L, HAYES G J, DICKEY M D, et al. Reconfigurable liquid metal circuits by Laplace pressure shaping[J]. Applied Physics Letters, 2012, 101(17): 277-303.

[26] CHUNG S, LEE J, SONG $\mathrm{H}$, et al. Inkjet-printed stretchable silver electrode on wave structured elastomeric substrate[J]. Applied Physics Letters, 2011, 98: 153110.

[27] 刘旭, 武澎, 吕延军. 一种柔性 PVDF 压电薄膜传感器 的制备方案 [J]. 仪表技术与传感器, 2016(1): 4-6.

LIU Xu, WU Peng, LÜ Yanjun. A preparation method of flexible PVDF piezoelectric thin film sensor[J]. Instrument Technique and Sensor, 2016(1): 4-6.

[28] 杨照光, 李志新, 杨军亭. 基于新型柔性压电薄膜传感 器的 GIS 故障准确定位系统的研究 $[\mathrm{J}]$. 电测与仪表, 2016, 53(14): 118-123.

YANG Zhaoguang, LI Zhixin, YANG Junting. Research on GIS fault location system based on nes flexible piezoelectric thin film sensor[J]. Journal of Electrical 
Tests and Instruments，2016，53(14): 118-123.

[29] 张悦. 多曲面 PVDF 力传感器的研究[D]. 大连: 大连理 工大学, 2014.

ZHANG Yue. Research on multi surface PVDF force sensor[D]. Dalian: Dalian University of Technology, 2014.

[30] 孙一心, 钟莹, 王向鸿. 柔性电容式触觉传感器的研究 与实验 $[\mathrm{J}]$. 电子测量与仪器学报, 2014, 28(12): 1394-1400.

SUN Yixin, ZHONG Ying, WANG Xianghong. Research and experiment of flexible capacitive tactile sensor[J]. Journal of Electronic Measurement and Instrument, 2014, 28(12): 1394-1400.

[31] 翟庆涁, 莫黎听, 杨威. 电容式柔性压力传感器性能影 响因素研究[J]. 信息记录材料, 2015, 16(5): 3-8. ZHAI Qingbin, MO Lixin, YANG Wei. Study on factors affecting the performance of capacitive flexible pressure sensors[J]. Information Recording Materials, 2015, 16(5): 3-8.

[32] 侯玉群. 柔性电容式压力传感器及其灵敏度影响因素 研究[D]. 北京: 北京印刷学院, 2015.

HOU Yuqun. Flexible capacitive pressure sensor and its sensitivity factors[D]. Beijing: Beijing Institute of Graphic Communication, 2015.

[33] 夏凯伦, 塞木强, 张荣荣. 纳米碳材料在可穿戴柔性导
电材料中的应用研究进展 $[\mathrm{J}]$. 物理化学学报, 2016, 32(10): 2427-2446.

XIA Kailun, JIAN Muqiang, ZHANG Yingying. Research progress in application of carbon nanomaterials in wearable flexible conductive materials[J]. Acta Physico-Chimica Sinica, 2016, 32(10): 2427-2446.

[34] MUTH J T, VOGT D M, TRUBY R L, et al. Embedded 3D printing of strain sensors within highly stretchable elastomers[J]. Advanced Materials, 2014, 26(36) : 6307-6312.

[35] XIA Y, WHITESIDES G M. Soft lithography[J]. Angewandte Chemie International Edition, 1998, 37(1): 550-575.

[36] CHOI D Y, KIM M H, OH Y S, et al. Highly stretchable, hysteresis-free ionic liquid-based strain sensor for precise human motion monitoring[J]. Acs Applied Materials \& Interfaces, 2017, 9: 1770-1780.

[37] MENGUC Y, PARK Y L, PEI H. Wearable soft sensing suit for human gait measurement[J]. The International Journal of Robotics Research, 2014, 33(14): 1748-1764.

作者简介: 姚建涛, 男 1980 年出生, 教授。主要研究方向为机器人机 构理论及多维力感知机构。

E-mail: jtyao@ysu. edu.cn

赵永生, 男, 1962 年出生, 教授, 博士研究生导师。主要研究方向为 机器人技术、传感器技术、先进制造技术。

E-mail: yszhao@ysu.edu.cn 